\title{
La clase magistral en el contexto del modelo educativo basado en competencias
}

\author{
The Master Class in the Context of the Competency-Based Educational Model
}

A aula magistral no contexto do modelo educacional baseado em competências

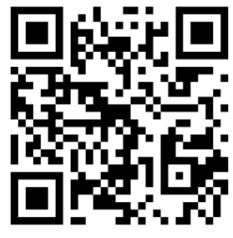

Mariela Gatica-Saavedra

Universidad San Sebastián

Facultad de Psicología

Concepción, Chile

magaticas@gmail.com

(iD) https://orcid.org/0000-0003-1657-3359

Patricia Rubí-González

Universidad de Concepción

Facultad de Medicina

Concepción, Chile

prubi@udec.cl

(iD https://orcid.org/0000-0002-5021-2605

Recibido • Received • Recebido: 02 / 05 / 2019

Corregido • Revised • Revisado: 01 / 11 / 2020

Aceptado • Accepted • Aprovado: 08 / 12/ 2020

\begin{abstract}
Resumen: El cambio del modelo curricular en educación superior hacia uno basado en competencias ha generado la búsqueda de metodologías que permitan la participación activa del estudiantado en el proceso enseñanza-aprendizaje. Así es como se han replanteado las metodologías utilizadas de manera tradicional, como la clase magistral. El objetivo de este artículo fue realizar un análisis crítico de la clase magistral a través de una revisión narrativa. Se recopiló el material utilizando las bases de datos Scielo, Web of Science y Google Académico, y en revistas de educación utilizando las palabras clave clase magistral, lección magistral, educación y en inglés master class y education, desde el año 2000 a 2018. Se encontraron, utilizando estas palabras clave: once artículos empíricos, dos artículos cualitativos, un metaanálisis, ocho revisiones, dos tesis y dos capítulos de libro. En relación con los principales hallazgos, se concluye que, pese a que la mayor parte de la evidencia desacredita la clase magistral, se proponen formas de optimizarla y no eliminarla, entendiendo que la combinación con otras estrategias potencia la efectividad del proceso de enseñanza-aprendizaje. Entre las implicancias de este estudio se señala la necesidad de no perder de vista que lo importante no sería la estrategia o metodología, sino que esta permita la adquisición de competencias necesarias para la adecuada inserción del estudiantado en el mundo laboral.
\end{abstract}

Palabras claves: Clase magistral; método de enseñanza; currículo por competencias; educación; educación universitaria. 
http://doi.org/10.15359/ree.25-1.17

http://www.una.ac.cr/educare

educare@una.ac.cr

\begin{abstract}
Changing the curricular model in higher education towards one based on competencies has generated the search for methodologies allowing students' active participation in the teachinglearning process. Thus, the methodologies used in a traditional way, such as the master class, have been rethought. This article aimed to conduct a critical analysis of the master class through a narrative review. The material was reviewed using the Scielo, Web of Science, and Google Academic databases; education journals were also examined using the Spanish keywords clase magistral (master class), lección magistral (master lesson), educación (education), and the English words master class and education, from 2000 to 2018. With these keywords, eleven empirical articles, two qualitative articles, a meta-analysis, eight reviews, two theses, and two book chapters were found. As main findings, it is concluded that, although most of the evidence discredits the master class, ways to optimize it and not eliminate it are proposed, understanding that the combination with other strategies enhances the effectiveness of the teaching-learning process. Among the implications of this study is the need to keep in mind that the important thing would not be the strategy or methodology, but that they allow the acquisition of necessary skills for the student to properly incorporate into the working world.
\end{abstract}

Keywords: Master class; teaching methods; competency-based education; education.

Resumo: A mudança do modelo curricular do ensino superior para um modelo baseado em competências tem gerado a busca de metodologias que permitam a participação ativa do corpo discente no processo de ensino-aprendizagem. É assim que as metodologias utilizadas de forma tradicional, como a aula magistral, têm sido repensadas. O objetivo deste artigo foi realizar uma análise crítica da aula magistral por meio de uma revisão narrativa. O material foi revisado nas bases de dados Scielo, Web of Science e Google Academic, e em periódicos de educação com as palavras-chave aula magistral, lição magistral, educação e em inglês master class e educação, de 2000 a 2018. encontraram, por meio das palavras-chave: onze artigos empíricos, dois artigos qualitativos, uma meta-análise, oito resenhas, duas teses e dois capítulos de livros. Em relação aos principais achados, conclui-se que apesar de que a maioria das evidências desacreditar a aula magistral, são propostas formas de otimizála e não elimina-la, entendendo que a combinação com outras estratégias potencializa a eficácia do processo ensino-aprendizagem. Entre as implicações deste estudo está a necessidade de não perder de vista que o importante não é a estratégia ou metodologia, mas sim que ela permite a aquisição de competências necessárias à adequada inserção dos estudantes no ambiente de trabalho.

Palavras-chave: Aula magistral; método de ensino; currículo por competências; educação; ensino superior.

\title{
Introducción
}

En la Conferencia Mundial sobre la Educación Superior en 1998, la Organización de las Naciones Unidas para la Educación, la Ciencia y la Cultura (UNESCO) plantea que una de las funciones y misiones de la educación superior es "reforzar la cooperación con el mundo del trabajo y el análisis y la previsión de las necesidades de la sociedad" (Organización de las Naciones Unidas para la Educación, la Ciencia y la Cultura [UNESCO], 1998, p. 8). En este contexto se plantea el modelo educativo basado en competencias como el modelo que permitiría dar respuesta a esta necesidad (Argudín Vázquez, 2005).

\footnotetext{
2 Mariela Gatica-Saavedra y Patricia Rubí-González
}

Los artículos de la Revista Electrónica Educare del Centro de Investigación y Docencia en Educación de la Universidad Nacional, Costa Rica, se comparten bajo términos de la Licencia Creative Commons: Reconocimiento, № Comercial, Sin Obra Derivada 3.0 Costa Rica. Las autorizaciones adicionales a las aquí delimitadas se pueden obtener en el correo: educare@una.c 
http://doi.org/10.15359/ree.25-1.17

El concepto de competencia se entiende como "saberes de ejecución" (Argudín Vázquez, 2005 , p. 3). El modelo educativo basado en competencias, por tanto, se entiende como una orientación que pretende dar respuesta a las necesidades laborales del mundo del trabajo, preparando al estudiantado para que llegue a desarrollar, con maestría, las habilidades que darán respuesta a esta necesidad (García-García et al., 2010).

El cambio del modelo curricular en educación superior en Chile, el cual se dirige al modelo por competencias (Schmal y Ruíz-Tagle, 2008), ha generado la búsqueda de metodologías que permitan la participación activa del estudiantado en el proceso de enseñanza-aprendizaje (Ballesta Pagán et al., 2011) y ha producido, también, un giro en el cual se le enseña al alumnado a aprender, adquiriendo tanto docentes como estudiantes nuevo roles en este proceso (Zúñiga Arbalti et al., 2017). En este contexto, se han replanteado las metodologías utilizadas de manera tradicional, como es el caso de la clase magistral.

La clase magistral tiene, por propósito, "presentar un tema mediante la revisión general de diferentes perspectivas, actualizar el conocimiento y describir los resultados incluyendo la experiencia para provocar en el estudiante la motivación a [explorar el contenido] en mayor profundidad (Pinilla, 2011 y Brown y Manogue, 2001)" (Domínguez et al., 2015, p. 514). Desempeña una función actualizadora, orientadora, metodológica y educativa (Serra Valdés, 2014). Sin embargo, puede ocurrir que, con esta estrategia, el estudiantado se vuelva un mero receptor de conocimiento (Krutakova, 2014). Por otra parte, la concepción del aprendizaje en esta práctica está basada principalmente en la memoria (Videla, 2010). Un estudio realizado en una escuela de medicina colombiana comparó las estrategias del aula invertida versus la clase magistral. Plantea que la clase magistral no puede ser la única estrategia de enseñanza utilizada en la carrera de medicina, ya que no permitirá al estudiantado estar preparado para las exigencias de su profesión ni para la adecuada aplicación del conocimiento adquirido (Domínguez et al., 2015).

Desde esta perspectiva, la clase magistral no daría respuesta al modelo por competencias, ya que presenta una actitud paternalista, en la cual el conocimiento ya viene dado. Sin embargo, el cambio a nuevos modelos implica cambios en la actitud, no solo del profesorado, sino también del alumnado, el que debe tomar una dinámica más participativa en la construcción de su aprendizaje (Domínguez et al., 2015).

La clase magistral se encuentra muy arraigada en la educación y es una de las prácticas pedagógicas tradicionales más difundidas (Sánchez-Carracedo y Barba Vargas, 2019). Dado que el conocimiento se renueva constantemente, la universidad debería estimular en el estudiantado la curiosidad, ayudarlo a optimizar sus habilidades de aprendizaje y estudio a través de la vida, además de una aplicación adecuada del conocimiento (Videla, 2010). 
http://doi.org/10.15359/ree.25-1.17

http://www.una.ac.cr/educare

educare@una.ac.cr

El objetivo de este artículo es realizar un análisis crítico a través de una revisión narrativa de la clase magistral y el rol que esta puede cumplir en el contexto de los nuevos cambios curriculares en educación superior.

\section{Método}

Se revisó el material utilizando los buscadores Scielo, Web of Science (WoS) y Google Académico y en revistas de educación mediante las palabras claves clase magistral, lección magistral, educación y en inglés master class y education. El periodo de búsqueda fue durante agosto y septiembre de 2018. A partir de esta revisión, se encontraron once artículos empíricos, dos artículos cualitativos, un metaanálisis, ocho revisiones, dos tesis y dos capítulos de libro. Se revisaron todos los documentos antes señalados, sin importar el tipo de estudio, con una metodología de revisión narrativa.

\section{Resultados}

Se entiende como un buen profesorado aquel que "mejor gestiona los escenarios y los medios para lograr el aprendizaje de sus alumnos" (Díaz Tremarias y Noriega Velásquez, 2009, p. 39). Se plantea que el trabajo del personal docente es de calidad cuando logra el cumplimiento de los objetivos propuestos a través de la organización y utilización de métodos que optimicen sus tiempos y los recursos con que cuenta, considerando en este proceso al estudiantado y la participación activa de estos (Krutakova, 2014). Desde esta perspectiva, la clase magistral no sería una mala estrategia, si es gestionada de manera adecuada.

Sin embargo, no existe evidencia que justifique el uso y permanencia de la clase magistral como estrategia pedagógica (Videla, 2010).

Las técnicas y las estrategias de enseñanza se entienden de manera diferente en el caso de las ciencias. Las estrategias de enseñanza y aprendizaje se entenderían como actividades conscientes e intencionales que guían determinadas metas de aprendizaje, es decir, se aplican de un modo intencional y deliberado a una tarea. Por otra parte, una técnica es marcadamente mecánica y rutinaria. Por lo tanto, las estrategias pueden contener varias técnicas. Sumado a lo anterior, la estrategia adopta, como criterio clasificador, el modelo educativo en el cual se aplica o del que forma parte. La estrategia por excelencia en el modelo tradicional es la lección o clase magistral, de uso habitual en el nivel universitario (Galiano y Sevillano García, 2015).

Otra mirada tiene relación con la mayor o menor participación del alumndo en el proceso de construcción del conocimiento. Desde esta perspectiva, existirían, entonces, metodologías tradicionales o pasivas que se centran en el personal docente y metodologías innovadoras, activas y participativas centradas en el alumnado y su aprendizaje activo. En la primera categoría se encontraría la clase magistral, mientras que en las metodologías activas 
http://doi.org/10.15359/ree.25-1.17

estarían, por ejemplo, el método de caso, el aprendizaje basado en problemas (ABP), el trabajo en grupos colaborativos, el portafolio y métodos que usan las tecnologías de la información y comunicación (Rodríguez Sánchez, 2011).

Un argumento a favor de estas nuevas técnicas tiene que ver con el rápido avance del conocimiento y en las demandas profesionales. En una propuesta planteada para estudiantes de medicina, las personas autoras sugieren que el alumnado debería recibir un marco en el que se pueda construir conocimiento a lo largo de toda una vida de aprendizaje (Prober y Khan, 2013). Desde esta perspectiva, la clase magistral no contribuiría a conseguir ese objetivo.

Sin embargo, con respecto a la instalación de nuevos métodos, también existen reparos. En un meta-análisis publicado recientemente se ha encontrado que la evidencia a favor del aula invertida versus el aprendizaje tradicional basado en conferencias no es concluyente, ya que los datos obtenidos en estudios previos presentan una alta heterogeneidad en relación con el método y a los análisis estadísticos utilizados, además del riesgo de sesgo en el estudio (Chen et al., 2018).

En un estudio realizado con tres escuelas de medicina de El Salvador para la enseñanza de anatomía, su personal docente prefería la clase magistral para enseñar contenidos teóricos y, para contenidos prácticos, técnicas grupales con supervisión, las que tampoco permiten al estudiantado gestionar su conocimiento. Por lo tanto, las estrategias grupales no necesariamente aseguran el desarrollo de la creatividad y la autonomía en el alumnado (Vasquez Flamenco, 2017).

Otro estudio sobre el aula invertida plantea que esta técnica requiere de un entrenamiento previo del profesorado en relación con el uso del tiempo y la orientación de la discusión. Debiera tener, también, la presencia de más docentes con distintos puntos de vista para ayudar a fomentar el aprendizaje (Domínguez et al., 2015). Lo antes señalado implicaría, por tanto, una inversión de recursos desde la universidad y un cambio de mentalidad desde el profesorado, ya que desde este modelo lo más importante sería poner al estudiantado en el centro del proceso de aprendizaje, fomentando en él la responsabilidad y la preparación adecuada del tema antes de llegar al aula. Lo anterior, para estimular la participación activa y la actitud crítica y reflexiva; se convertiría, así, el profesorado en un facilitador del aprendizaje y no solo en un transmisor de información (Domínguez et al., 2015).

La clase magistral, en comparación con métodos más aplicados, tendría ventajas y desventajas. Entre las desventajas del currículo sofisticado, este podría tener un nivel cuestionable de costo-efectividad y limita la capacidad de validar el aprendizaje frente al avance del conocimiento y la nueva evidencia, en oposición a la clase magistral. Por otra parte, el currículo basado en las necesidades fomenta la resolución de problemas con los recursos disponibles y mantiene un enfoque vanguardista. Proponen, por tanto, un currículo en contexto, el cual iría a la delantera del conocimiento, al considerar los problemas y necesidades específicas de aprendizaje (Domínguez et al., 2015). 
http://doi.org/10.15359/ree.25-1.17

http://www.una.ac.cr/educare

educare@una.ac.cr

Otras propuestas se refieren a la optimización de la clase magistral. Según Herrán (2011, citado en Zúñiga-Escobar, 2017), para que la clase magistral sea efectiva debería ser motivadora, amena, respetuosa y con una extensión e intensidad adecuada a la curva media de concentración del estudiantado, y estar vinculada a otras actividades didácticas. Se propone, por tanto, que la clase magistral siempre se asuma como punto de partida y no como desembocadura, "como técnica inicial durante el abordaje de la intención educativa, pues implica una base teóricoconceptual para orientar los procedimientos prácticos siguientes"(Zúñiga-Escobar, 2017, p. 3).

Sánchez-Carracedo y Barba Vargas (2019) plantean que las clases magistrales serían apropiadas para la enseñanza, debido a que permiten aplicarlas a grupos numerosos de manera económicamente rentable, controlar mejor el tiempo y el contenido que se va a trabajar en clase, la estructura de esta y la dinámica utilizada, pero no sería tan apropiada para el proceso del aprendizaje, por esta razón proponen, también, formas de optimizarla considerando la neurociencia.

En la enseñanza de la medicina, se plantea que una forma de optimizar la clase magistral sería complementándola a través del uso de minicasos clínicos, los que podrían ayudar a aumentar el interés de parte del estudiantado. Debido a diversas razones, como las económicas, las logísticas o las psicológicas, es difícil pensar que este método desaparezca en un futuro próximo y sería conveniente buscar alternativas que permitan dinamizarla de manera que sobrepase la transmisión pasiva de información (Baños y Farré, 2011).

En el caso de la enseñanza de la bioética, inicialmente se comprobó que la clase magistral no era efectiva por lo monótona, por lo que se privilegió la enseñanza práctica en grupos pequeños. Sin embargo, esta metodología también ha sido desacreditada, debido a que tiende a perder el orden sistemático, deja temas sin tratar o gasta mucho tiempo en otras cosas. Por esta razón, se considera que la combinación de clases teóricas con actividades prácticas sería la mejor estrategia para la transmisión teórica de la bioética enriquecida por el ejercicio práctico (Kottow, 2009).

Algunas propuestas con metodología mixta han presentado también buenos resultados en la enseñanza de fisiología y anatomía. En estas se incorpora la clase magistral, la cual sería efectiva en la transmisión de la información, en conjunto con actividades realizadas en grupo y prácticas, las cuales potencian la integración de los conocimientos adquiridos durante la lección magistral. Este modelo mixto favorecería la estabilización del aprendizaje a un nivel suficiente, permitiendo equilibrar las diferencias individuales (Gal-Iglesias et al., 2009).

Otro aspecto relacionado con la estrategia a utilizar es la carrera. En el caso de la carrera de derecho, la clase magistral meramente expositiva continúa siendo recurrente en la formación jurídica. Sin embargo, se propone evolucionar a una clase magistral trascendente o formativa, en la cual el alumnado tome un papel más activo e interesado, desplazando al profesorado experto. Este tipo de docencia se centraría más en el proceso de enseñanza-aprendizaje, a 
http://doi.org/10.15359/ree.25-1.17

diferencia de la clase meramente expositiva que solo se centraría en la enseñanza (Elgueta Rosas y Palma González, 2014).

En un estudio cualitativo se conceptualizó la clase magistral universitaria como una actividad que es simultáneamente interacción y un tipo de discurso dialógico. La tarea de transmitir al estudiantado información compleja organizada desde la posición de personal experto tiene el objetivo de facilitar el aprendizaje de las condiciones y procedimientos que los sujetos receptores-destinatarios deben aplicar para adquirir las competencias propias de cada materia. Interpretar la situación expositiva como interlocución transaccional desde la posición docente es el enfoque que puede transformar el patrón "los estudiantes siguen al docente" en un intercambio cooperativo alimentado por el comportamiento del público oyente-destinatario y canalizado por el sujeto hablante-destinador (Tronchoni et al., 2018).

Otro aspecto importante de considerar es el estilo de aprendizaje del alumnado. Se plantea que cuando no se identifican los estilos de aprendizaje y se establecen relaciones entre los procesos vinculados con el aprendizaje, o estos se estudian de manera independiente, los procesos suelen ser de poca utilidad para la práctica docente. Por lo tanto, no solo sería necesario conocer la técnica de enseñanza que utiliza el profesorado, sino la forma en que aprende el alumnado. Según esto, el interés debería centrarse en la forma en cómo el personal docente podría aportar al proceso de enseñanza-aprendizaje desde la incorporación de ajustes metodológicos a partir de los estilos de aprendizaje según lo que pretenda lograr en el estudiantado. En un estudio realizado con estudiantes de la carrera de kinesiología en Antofagasta (Chile) se encontró que los estilos de aprendizaje evolucionan según el curso. El alumnado de tercer año presentaba preferentemente estilos teórico y pragmático, en tanto que en el alumnado de cuarto y quinto año estilos activo y reflexivo. A partir de este hallazgo, la clase magistral sí podría ser útil (Solari-Montenegro et al., 2017).

Lo anterior se puede relacionar con el concepto de aprendizaje significativo de Ausubel (de la Herrán, 2011). El autor plantea que el aprendizaje depende de las relaciones entre el conocimiento nuevo y que ya se posee, y de la intención consciente de realizar conexiones entre ellas. Por lo tanto, el conocimiento que no es conectado, que es el que se tiende a memorizar, no se puede usar para razonar o resolver problemas en nuevos contextos (Videla, 2010). Si la clase magistral está de acuerdo con los intereses y conocimientos del estudiantado, puede ser una técnica generadora de actividad intelectual y puede tener una buena capacidad formativa (Martín y Solé, 2008). Desde la mirada de Ausubel, se puede favorecer el aprendizaje significativo por recepción a través de una buena exposición, ya que el aprendizaje significativo no se da solo por descubrimiento. Se plantea que, si todo lo que hay que conocer durante nuestra existencia debemos aprenderlo descubriendo nuevamente, manipulando o experimentando, no se alcanzaría a saber todo lo que se debe saber. Ello podría afectar al avance de la ciencia y la tecnología (Charaja Cutipa, 2014; Ríos Pezo, 2015; Ward y Barry, 2018). 
http://doi.org/10.15359/ree.25-1.17

http://www.una.ac.cr/educare

educare@una.ac.cr

Con respecto a la actitud del alumnado hacia la clase magistral, este valora la interacción estudiante-docente y la prefiere por sobre la educación a distancia, debido a que permite el diálogo y enriquece la discusión; sin embargo, prefiere que a esta se incorporen otros medios audiovisuales como complemento, lo cual contribuiría a optimizar el aprendizaje (Díaz Tremarias y Noriega Velásquez, 2009).

Finalmente, la clase magistral se ha usado también como refuerzo en aquellas falencias en la formación o bien para apoyar al estudiantado que percibe que puede tener estas dificultades; así cumple, por tanto, además de un rol formativo, una forma de contribución a entregar confianza y motivación al alumnado con respecto al rol que puede desempeñar en el ejercicio de su profesión (Charaja Cutipa, 2014).

\section{Conclusión}

Teniendo como antecedente el cambio curricular hacia un modelo basado en competencias, las estrategias de enseñanza debiesen estar orientadas a desarrollar y formar a un alumnado más activo en el rol de adquisición del aprendizaje. Lo anterior, para habilitar de mejor manera al estudiantado y permitir su mejor inserción en el mundo laboral y responder, de mejor manera, a sus demandas. Desde esta mirada, no habría una metodología mejor que otra, ya que cualquier metodología sería adecuada, si esta se ajusta a las características de los aprendizajes, la finalidad que se persigue en función de las competencias que se busca desarrollar, si el uso técnico es correcto y si no se utilizan de manera reactiva unas frente a otras (Rodríguez Sánchez, 2011).

La mayoría de los documentos analizados en esta revisión proponen nuevas metodologías más activas, en desmedro de la clase magistral; sin embargo, se observa también que esta puede aplicarse de manera combinada, con resultados exitosos y optimizando el proceso de enseñanza-aprendizaje.

La implementación de metodologías más activas implica un cambio de mentalidad a nivel de docentes, de estudiantes y de las mismas universidades. Por esta razón, el cambio de una estrategia a otra probablemente será progresivo. Mientras eso ocurre, es conveniente, por tanto, informarse en relación con las técnicas que pudieran optimizar la clase magistral y permitan complementarla con estrategias más participativas.

Si se tienen en consideración factores como el tipo de aprendizaje del alumnado, el ramo que se enseña y la carrera que se imparte, la clase magistral puede mantenerse integrada en el currículo actual. Si esta está dirigida a estimular la motivación por continuar conociendo, su rol educativo se estaría cumpliendo, pues a partir de ella, también se puede generar aprendizaje 
http://doi.org/10.15359/ree.25-1.17

significativo. Es importante no perder de vista que no es la metodología en sí lo importante en el modelo educativo basado en competencias, o el hecho de que ciertas metodologías o estrategias sean específicas a este, sino que estas deben dar respuesta a la adquisición de las competencias necesarias para una adecuada inserción al mundo laboral.

\section{Referencias}

Argudín Vázquez, Y. (2005). Educación basada en competencias. http://www.quadernsdigitals.net/ index.php?accionMenu=hemeroteca.DescargaArticulolU.descarga\&tipo=PDF\&articulo $\underline{i d=7587}$

Ballesta Pagán, F. J., Izquierdo Rus, T. y Romero Sánchez, B. E. (2011). Percepción del alumnado de pedagogía ante el uso de metodologías activas. Educatio Siglo XXI, 29(2), 353-368. https:// revistas.um.es/educatio/article/view/133101/122801

Baños, J. E. y Farré, M. (2011). Dinamización de la clase magistral en medicina: Diez ejemplos de minicasos utilizados en la docencia de la farmacología. Educación Médica, 14(2), 105-112. https://doi.org/10.4321/S1575-18132011000200006

Charaja Cutipa, F. (2014). Vigencia de la clase magistral en la universidad del siglo XXI. Apuntes Universitarios. Revista de investigación, 4(1), 57-66. https://doi.org/10.17162/au.v0i1.51

Chen, K.-S., Monrouxe, L., Lu, Y.-H., Jenq, C.-C., Chang, Y.-J., Chang, Y.-C. y Chai, P. Y.-C. (2018). Academic outcomes of flipped classroom learning: A meta-analysis. Medical Education, 52(9), 910-924. https://doi.org/10.1111/medu.13616

Martín, E. y Solé, I. (2008). El aprendizaje significativo y la teoría de la asimilación. En C. Coll, J. Palacios y A. Marchesi (Comps.), Desarrollo psicológico y educación. 2. Psicología de la educacion escolar ( $2^{\circ}$ ed., Vol. 2, pp. 89-114). Alianza Editorial. https://www. academia.edu/43352606/Desarrollo psicol\%C3\%B3gico y educaci\%C3\%B3n 2 Psicolog\%C3\%ADa de la educaci\%C3\%B3n

de la Herrán, A. (2011). Técnicas didácticas para una enseñanza más formativa". En N. Álvarez y R. Cardoso (Coords.), Estrategias y metodologías para la formación del estudiante en la actualidad. Universidad de Camagüey. 
http://doi.org/10.15359/ree.25-1.17

http://www.una.ac.cr/educare

educare@una.ac.cr

Díaz Tremarias, M. y Noriega Velásquez, T. (2009). Utilización de videos didácticos como innovación en la enseñanza de la toxicología. Educación Médica Superior, 23(3), 38-44.

Domínguez, L. C., Vega, N. V., Espitia, E. L., Sanabria, Á. E., Corso, C., Serna, A. M. y Osorio, C. (2015). Impacto de la estrategia de aula invertida en el ambiente de aprendizaje en cirugía: Una comparación con la clase magistral. Biomédica, 35(4), 513-521. https://doi.org/10.7705/ biomedica.v35i4.2640

Elgueta Rosas, M. F. y Palma González, E. E. (2014). Una propuesta de clasificación de la clase magistral impartida en la facultad de derecho. Revista chilena de derecho, 41(3), 907-924. https://doi.org/10.4067/S0718-34372014000300006

Galiano, J. E. y Sevillano García, M. L. (2015). Estrategias de enseñanza de la química en la formación inicial del profesorado universitario. Educatio Siglo XXI, 33(1), 215-234. https:// doi.org/10.6018/j/222571

Gal-Iglesias, B., Busturia-Berrade, I. y Garrido-Astray, M. C. (2009). Nuevas metodologías docentes aplicadas al estudio de la fisiología y la anatomía: Estudio comparativo con el método tradicional. Educación Médica, 12(2), 117-124. https://doi.org/10.4321/S157518132009000300008

García-García, J. A., González-Martínez, J. F., Estrada-Aguilar, L. y Uriega-González-Plata, J. S. (2010). Educación médica basada en competencias. Revista Médica del Hospital General de México, 73(1), 57-69. https://www.medigraphic.com/cgi-bin/new/resumen. cgi?IDARTICULO=24412

Kottow, M. H. (2009). Enseñanza de la bioética: Una síntesis. Revista Brasileira de Educação Médica, 33(4), 658-663. https://doi.org/10.1590/S0100-55022009000400017

Krutakova, A. (2014). Métodos docentes para la enseñanza-aprendizaje de la economía. Aplicación a la unidad didáctica "El dinero y el sistema financiero" [Tesis de maestría]. Universidad de Valladolid, España. http://uvadoc.uva.es/handle/10324/10382

Organización de las Naciones Unidas para la Educación, la Ciencia y la Cultura. (1998). Declaración mundial sobre la educación superior en el siglo XXI: Visión y acción preámbulo. https:// www.oei.es/historico/salactsi/DECLARACION MUNDIAL EDUCACION SUPERIOR.pdf

Prober, C. G. y Khan, S. (2013). Medical education reimagined: A call to action. Academic Medicine, 
http://doi.org/10.15359/ree.25-1.17

http://www.una.ac.cr/educare educare@una.ac.cr

88(10), 1407-1410. https://doi.org/10.1097/ACM.0b013e3182a368bd

Ríos Pezo, M. (2015). Influencia del seminario y la clase magistral en el rendimiento académico de alumnos de las facultades de medicina humana UNAP 2015 [Tesis de doctorado]. Universidad Nacional de la Amazonía Peruana, Iquitos, Perú. http://repositorio.unapiquitos.edu.pe/ handle/UNAP/3952

Rodríguez Sánchez, M. (2011). Metodologías docentes en el EEES: De la clase magistral al portafolio. Tendencias pedagógicas, 17, 83-103. https://dialnet.unirioja.es/servlet/ articulo?codigo $=3653734$

Sánchez-Carracedo, F. y Barba Vargas, A. (2019). Cómo impartir una clase magistral según la neurociencia. Actas de las Jenui, 4, 87-94. http://hdl.handle.net/2117/166394

Schmal, R. y Ruíz-Tagle, A. (2008). Una metodología para el diseño de un currículo orientado a las competencias. Ingeniare. Revista Chilena de Ingeniería, 16(1), 147-158. https://doi. org/10.4067/S0718-33052008000100004

Serra Valdés, M. Á. (2014). Reflexiones sobre metodología y didáctica de impartición de la conferencia en el ciclo clínico de la Educación Médica Superior. Educación Médica Superior, 28(3), 456-466. http://scielo.sld.cu/scielo.php?script=sci arttext\&pid $=$ S0864-21412014000300007

Solari-Montenegro, C. G., Rivera-Iratchet, M. E. y Velasco-Mur, A. (2017). Prevalencia de estilos de aprendizaje de los estudiantes de primer semestre de tercero, cuarto y quinto año, y de estilos docentes de la carrera de Cinesiología, Universidad de Antofagasta. Revista de la Fundación Educación Médica, 20(2), 57-64. https://doi.org/10.33588/fem.202.880

Tronchoni, H., Izquierdo, C. y Anguera, M. T. (2018). Interacción participativa en las clases magistrales: Fundamentación y construcción de un instrumento de observación. Publicaciones, 48(1), 77-95. https://doi.org/10.30827/publicaciones.v48i1.7331

Vasquez Flamenco, G. (2017). Estrategias y técnicas didácticas de la relación numérica docente - estudiante en anatomía humana. Revista médica electrónica, 39(4), 894-905. http://scielo. sld.cu/scielo.php?script=sci abstract\&pid=S1684-18242017000400005

Videla, R. L. (2010). Clases pasivas, clases activas y clases virtuales. ¿Transmitir o construir conocimientos? Revista Argentina de Radiología, 74(2), 187-191. https://www.redalyc.org/ 
http://doi.org/10.15359/ree.25-1.17

http://www.una.ac.cr/educare

educare@una.ac.cr

articulo.oa?id=382538482010

Ward, L. y Barry, S. (2018). The mental health master class: An innovative approach to improving student learning in mental health nursing. International Journal of Mental Health Nursing, 27(5), 1-10. https://doi.org/10.1111/inm.12450

Zúñiga Arbalti, F. A., Castillo Suazo, S., Aguayo Tapia, C., Sánchez Ramos, O., Salas Burgos, A., Hernández Montes, L. y Ormazábal Valladares, V. A. (2017). Utilización de aprendizaje basado en equipos, como metodología activa de enseñanza de farmacología para estudiantes de enfermería. Educación Medica Superior, 31(1), 78-88. http://scielo.sld.cu/ scielo.php?script=sci arttext\&pid=S0864-21412017000100008

Zúñiga-Escobar, M. (2017). La estrategia didáctica: Una combinación de técnicas didácticas para desarrollar un plan de gestión de riesgos en la clase. Revista Educación, 41(1), 1-18. https:// doi.org/10.15517/revedu.v41i1.17786 